\title{
On Optimal Realizations of Finite Metric Spaces by Graphs
}

\author{
Ingo Althöfer \\ Fakultät für Mathematik, Universität Bielefeld, Postfach 8640, D-4800 Bielefeld, \\ Federal Republic of Germany
}

\begin{abstract}
Graph realizations of finite metric spaces have widespread applications, for example, in biology, economics, and information theory. The main results of this paper are:

1. Finding optimal realizations of integral metrics (which means all distances are integral) is NP-complete.

2. There exist metric spaces with a continuum of optimal realizations.

Furthermore, two conditions necessary for a weighted graph to be an optimal realization are given and an extremal problem arising in connection with the realization problem is investigated.
\end{abstract}

\section{Introduction}

To find graph realizations of metrics or distance matrices is an area of research which has been given much attention (see [1]-[16] and [18]-[21]). The subject has widespread applications, perhaps most interesting is its use in a biological model to reconstruct phylogenetic trees from matrices, whose entries represent certain genetic distances among contemporary living species.

In this paper we present several new results on the topic. Section 2 supplies two local conditions (redundant edges, redundant edge pairs) necessary for optimal realizations, which are helpful tools in checking the optimality of a given realization. In Section 3 the optimal realization problem for integral metrics (which means all distances are integral) is shown to be NP-hard, while Section 4 supplies its NP-membership. Hence a quick (or at least a polynomial) algorithm for the realization problem is very unlikely to exist. Section 5 contains, beside other examples, a metric with a continuum of optimal realizations, which disproves a conjecture of Dress [5] and gives rise to an interesting new question. Section 6 investigates extremal quotients of total edge lengths in optimal realizations and total distances in the underlying metric $(M, d)$, where $|M|=m$ is fixed. 
The infimum for these quotients is shown to be $4 / m^{2}$ if $m$ is even, and $4 /\left(m^{2}-1\right)$ if $m$ is odd. The supremum is bounded from below by $\frac{1}{3}$ and from above by 1 for all $m \geq 1$.

Let $G=G(V, E, w)$ be a finite undirected simple graph with vertex set $V$, edge set $E$, and $w: E \rightarrow \mathbb{R}^{>0}$ a function which assigns a positive weight or length to every edge of $G$. Let $d_{G}(x, y)$ denote the length of a shortest path from vertex $x$ to vertex $y$ in $G$. The weighted graph $G$ realizes a finite metric $(M, d)$ if $M \subset V$ and $d(i, j)=d_{\sigma}(i, j)$ for all $i, j$ of $M$.

The elements in $V-M$ are called auxiliary vertices of the realization. A realization $G(V, E, w)$ of $(M, d)$ is optimal if $\sum_{e \in E} w(e)$ is minimal among all realizations of $(M, d)$.

Recall the following known results:

Theorem A [5], [14]. Every finite metric $(M, d)$ has an optimal realization.

Theorem B [5, p. 396]. For every optimal realization $G(V, E, w)$ of a metric $(M, d)$ with $|M|=m$

$$
|\{v \in V \mid \operatorname{deg} v>2\}| \leq \frac{m(m-1)^{2}(m-2)}{4} \leq m^{4}
$$

Theorem $C$ [14]. Let $G=G(V, E, w)$ be a realization of $(M, d)$ with $M=V$. Then $G$ is the unique optimal realization of $(M, d)$ iff the following two conditions are satisfied:

(a) $d(x, z)=d(x, y)+d(y, z)$ for all edges $\{x, y\}$ and $\{y, z\}$ of $G$.

(b) $d(x, y)+d(t, z)<\max \{d(x, t)+d(y, z), d(x, z)+d(y, t)\}$ for all edges $\{x, y\},\{t, z\}$ with no common endpoints.

Theorem $\mathbf{D}[9]$. If a metric has a tree realization, this realization is unique and optimal.

Theorem E [7, p. 194]. The problem of finding maximal independent sets in graphs without triangles and with all vertices having degree at least 2 is NP-complete.

\section{Necessary Local Conditions for Optimal Realizations}

The following statement is well known (see [18]).

Lemma 2.1. Every optimal realization $G(V, E, w)$ of a metric $(M, d)$ satisfies the following two conditions:

(a) For every $e \in E$ there exist $i, j \in M$, such that every shortest path from $i$ to $j$ in $G$ contains $e$.

(b) Let $e, e^{\prime} \in E$ be two edges with a common endpoint in $G$. Then there exist $i$, $j \in M$, such that at least one shortest path from $i$ to $j$ contains the edge sequence ee'. 


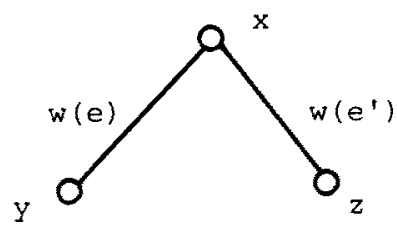

Fig. 1

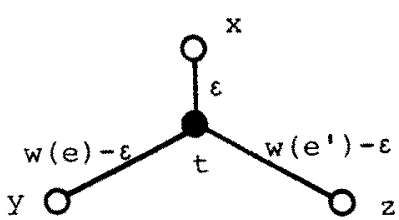

Fig. 2

Proof. Assume that (a) is not valid for some $e \in E_{y}$ then by deleting $e$ from $G$ obtain another realization of $(M, d)$ with a smaller total length.

Similarly, if (b) fails for some ee' with a common vertex $x$, find another realization of $(M, d)$ with a smaller total length by replacing the subgraph in Fig. 1 with that in Fig. 2 for some small $\varepsilon>0$ and an additional auxiliary vertex $t$.

Definition 2.2. Let $(M, d)$ be a metric with realization $G(V, E, w)$. $G$ is called minimal, if:

(a) $G$ contains no edge $e$ such that $G-e$ realizes $M$ (redundant edge).

(b) $G$ contains no edge pair $e e^{\prime}$ with common vertex such that every shortest path with endpoints in $M$ omits at least one of $e, e^{\prime}$ (redundant edge pair).

Remark 2.3. Lemma 2.1 states that every optimal realization is minimal. The inverse does not hold as may be seen by the following example.

Example 2.4. The graphs $G_{1}\left(V_{1}, E_{1}, w_{1}\right)$ in Fig. 3 and $G_{2}\left(V_{2}, E_{2}, w_{2}\right)$ in Fig. 4

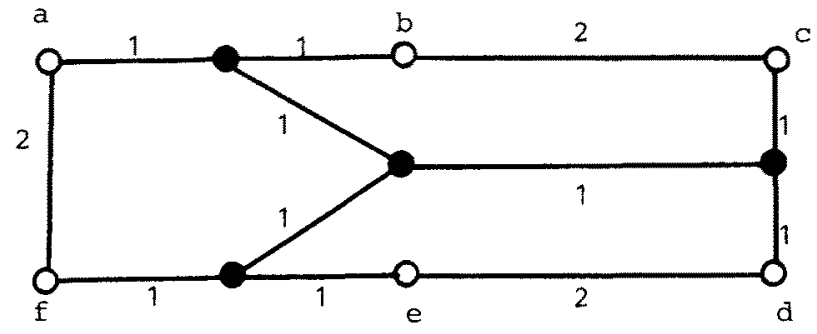

Fig. 3

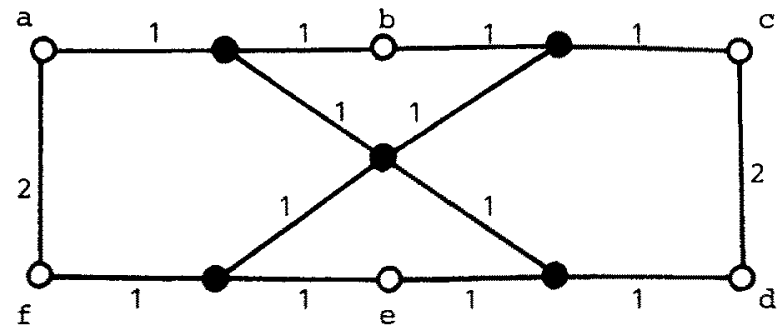

Fig. 4 
are both minimal realizations of the same metric $d$ on $M:=\{a, b, c, d, e, f\} . G_{2}$ is not optimal as $\sum_{e \in E_{2}} w_{2}(e)=16 \geq 15=\sum_{e \in E_{1}} w_{1}(e)$.

Remark 2.5. By going through the proof $[5$, p. 396] it may be observed that Theorem $B$ is valid for realizations without redundant edges and not only for the more special optimal realizations. So, in particular, it holds for minimal realizations.

\section{Searching for Optimal Realizations is NP-Hard}

The realization problem has a simple solution for tree realizable metrics [17]. On the other hand, no good algorithm is known for arbitrary metrics, even with integral distances only. That this problem is NP-hard, follows from Theorem 3.2 below. This theorem reduces the search for minimal transversals in certain graphs to the construction of optimal realizations for corresponding metrics.

Definition 3.1. Let $G(V, E)$ be a simple, undirected graph. A transversal $T$ is a subset of $V$, such that each edge has at least one endpoint in $T$.

Thus transversals are the complements of independent sets. Finding maximal independent sets is known to be NP-hard. Theorem E states this result for the graph class examined in Theorem 3.2.

For every unweighted graph $G(V, E)$ with transversal $T$ the following construction supplies a weighted graph $H_{T}(\bar{V}, \bar{E}, \bar{w})$.

Construction. Subdivide every edge $e$ of $G$ by a new vertex " $e$." Include a new "central point" $z$ and connect it with all vertices in $T$. Assign length $\frac{1}{2}$ to all edges in the resulting graph $H_{T}$.

Figure 5 shows $H_{T}(\bar{V}, \bar{E}, \bar{w})$ for the 4-cycle with vertices $\left\{v_{1}, v_{2}, v_{3}, v_{4}\right\}$ and $T=\left\{v_{1}, v_{3}\right\}$.

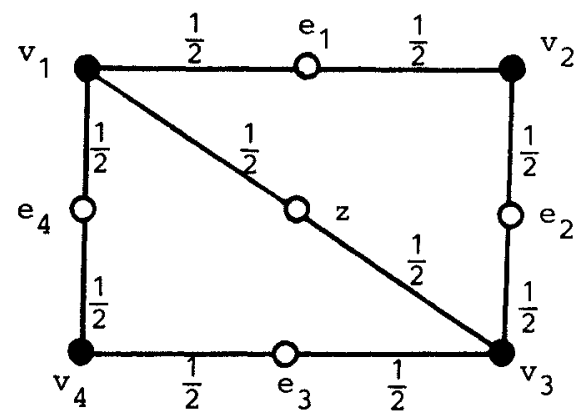

Fig. 5 
In general, $H_{T}$ induces a metric $\left(M_{G}, d\right)$ on $M_{G}=E \cup\{z\}$

$$
d(x, y)= \begin{cases}0 & \text { if } x=y \\ 1 & \text { if } z \in\{x, y\}, x \neq y \\ 1 & \text { if } x, y \text { are edges in } G \text { with a common endpoint, } x \neq y \\ 2 & \text { otherwise. }\end{cases}
$$

Observe that $\left(M_{G}, d\right)$ does not depend on $T$.

Theorem 3.2. Let $G(V, E)$ be a simple undirected unweighted graph without triangles and with degree at least 2 for all vertices. Let $T \subset V=\{1, \ldots, n\}$ be $a$ minimal transversal in $G$. Then $H_{T}(\bar{V}, \bar{E}, \bar{w})$ is an optimal realization of $\left(M_{G}, d\right)$ and has total edge length $|E|+\frac{1}{2}|T|$.

The conditions "without triangles" and "deg $\geq 2$ " are necessary, as is shown by two examples. The underlying graphs are the complete graphs on three vertices (Figs. 6 and 7) and on two vertices (Figs. 8 and 9). All edges have length $\frac{1}{2}$.

Proof of Theorem 3.2. Obviously $H_{T}$ has total edge length $|E|+\frac{1}{2}|T|$. So it remains to show that no other realization has a smaller total length. For this let $\tilde{H}(\tilde{V}, \tilde{E}, \tilde{w})$ be an arbitrary minimal realization of $\left(M_{G}, d\right)$. Without loss of generality let $\operatorname{deg} x \geq 3$ for all $x \in \tilde{V}-M_{G}$. $\tilde{E}$ is partitioned by

$$
\begin{aligned}
& \tilde{E}^{\prime}:=\left\{\tilde{e} \in \tilde{E} \mid \tilde{e} \text { lies on a shortest path from } e_{1} \text { to } e_{2} \text { for some } e_{1}, e_{2} \in E\right. \\
& \text { with a common endpoint in } \left.G \text { and thus } d\left(e_{1}, e_{2}\right)=1\right\}, \\
& \tilde{E}^{\prime \prime}:=\tilde{E}-\tilde{E}^{\prime} .
\end{aligned}
$$

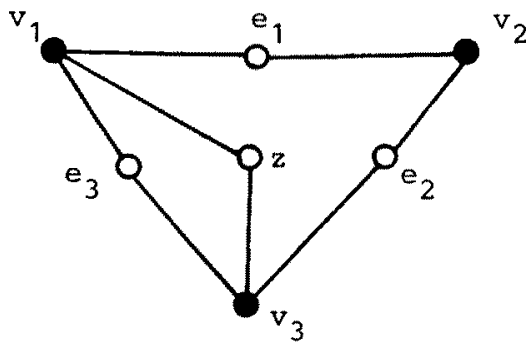

Fig. 6

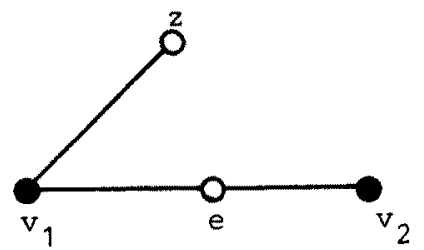

Fig. 8

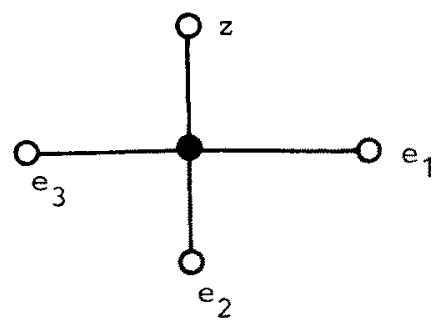

Fig. 7

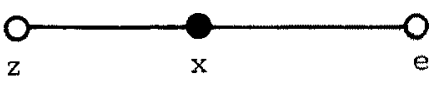

Fig. 9 
Lemma 3.3 below yields $\sum_{\tilde{e}_{\in} \tilde{E}} \tilde{w}(\tilde{e}) \geq|E|$, in Lemma $3.4 \sum_{\tilde{e}_{E} \tilde{E}^{n}} \tilde{w}(\tilde{e}) \geq \frac{1}{2}|T|$ is shown, thus altogether $\sum_{\tilde{e} \in \tilde{E}} \tilde{W}(\tilde{e}) \geq|E|+\frac{1}{2}|T|$.

Lemma 3.3. A partition $\tilde{E}^{\prime}=\tilde{E}_{i}^{\prime} \cup \cdots \dot{\cup} \tilde{E}_{n}^{\prime}$, all $\tilde{E}_{i}^{\prime} \neq \varnothing$, is given by

$$
\begin{aligned}
& \tilde{E}_{i}^{\prime}:=\left\{\tilde{e} \in \tilde{E}^{\prime} \mid \tilde{e} \text { lies on a shortest path between } e_{1}, e_{2} \in E\right.\text { with a common endpoint } i \text { in } G\}, \\
& \sum_{\tilde{e} \in \tilde{E}^{\prime}} \tilde{w}(\tilde{e})=\sum_{i=1}^{n} \sum_{\tilde{e} \in \tilde{E}_{i}} \tilde{w}(\tilde{e}) \geq \sum_{i \in V} \frac{1}{2} \operatorname{deg} i=|E| .
\end{aligned}
$$

Proof of Lemma 3.3. Obviously $\tilde{E}^{\prime}=\bigcup_{i=1}^{n} \tilde{E}_{i}^{\prime}$ and $\tilde{E}_{i}^{\prime} \neq \varnothing$ for all $i \in V$, as every vertex $i$ has degree at least 2 . The disjointness is proved by contradiction. Assume $i, j \in V, i \neq j$, and $\tilde{e} \in \tilde{E}_{i}^{\prime} \cap \tilde{E}_{j}^{\prime}$. Hence there are $e_{1}, e_{2} \in E$ with a common endpoint $i \in V$ and $e_{3}, e_{4} \in E$ with a common endpoint $j \in V$ in $G$, such that a shortest path from $e_{1}$ to $e_{2}$, and also a shortest path from $e_{3}$ to $e_{4}$, in $\tilde{H}$ contains $\tilde{e}$. The set $A=\left\{e_{1}, e_{2}, e_{3}, e_{4}\right\}$ has at least three elements, since otherwise, for example, $e_{1}=e_{3}, e_{2}=e_{4}$, and $\left\{e_{1}, e_{2}\right\}$ would be a double edge between $i$ and $j . G$ does not contain any triangle. Hence there are $e, e^{\prime} \in A$ with no common endpoint in $G$. Without loss of generality $e=e_{1}, e^{\prime}=e_{4}$. Therefore

$$
d\left(e_{1}, e_{4}\right)=d_{\tilde{H}}\left(e_{1}, e_{4}\right)=2 .
$$

Let $\tilde{e}=\left\{z_{1}, z_{2}\right\}$.

Case 1. $e_{1}-\cdots-z_{1}-z_{2}-\cdots-e_{2}$ is a shortest path from $e_{1}$ to $e_{2}, e_{3}-\cdots-z_{1}-$ $z_{2}-\cdots-e_{4}$ is a shortest path from $e_{3}$ to $e_{4} . d_{\tilde{H}}\left(e_{1}, z_{2}\right) \leq 1, d_{\tilde{H}}\left(z_{2}, e_{4}\right)<1$. Hence $d_{\tilde{H}}\left(e_{1}, e_{4}\right)<2$, which contradicts $(1)$.

Case 2. $e_{1}-\cdots-z_{1}-z_{2}-\cdots-e_{2}$ and $e_{4}-\cdots-z_{1}-z_{2}-\cdots-e_{3}$ are shortest paths from $e_{1}$ to $e_{2}$ and $e_{4}$ to $e_{3} . d_{\tilde{H}}\left(e_{1}, z_{1}\right)<1, d_{\tilde{H}}\left(z_{1}, e_{4}\right)<1$. Hence $d_{\tilde{H}}\left(e_{1}, e_{4}\right)<2$, again contradicting (1).

In the partial graph $\tilde{H}_{i}\left(\tilde{V}, \tilde{E}_{v}^{\prime}, \tilde{w}\right)$ of $\tilde{H}$ all pairs $e_{1}, e_{2} \in E$ with a common endpoint $i$ in $G$ have distance 1 . Hence $\tilde{H}_{i}$ realizes a metric $\left(B_{i}, d_{1}\right)$, where $B_{i}=\{e \in E \mid i$ is an endpoint of $e$ in $G\}$ and $d_{i}\left(e_{1}, e_{2}\right)=1$ for all $e_{1} \neq e_{2} \in B_{i}$. The star in Fig. 10 realizes $\left(B_{i}, d_{i}\right)$ with a total length $\frac{1}{2} \mathrm{deg} i$. Center $c$ is an auxiliary vertex. By Theorem $\mathrm{D}$ this tree is the optimal realization of $\left(B_{1}, d_{1}\right)$. Thus $\sum_{\tilde{e} \in \tilde{E}_{i}} \tilde{w}(\tilde{e}) \geq \frac{1}{2} \operatorname{deg} i$, which completes the proof of Lemma 3.3.

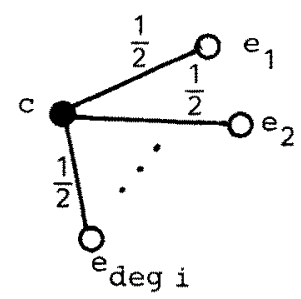

Fig. 10 
$\left(M_{G}, d\right)$ has a special structure with distances 1 and 2 only and $d\left(e_{1}, e_{2}\right)=$ $d\left(e_{1}, z\right)+d\left(z, e_{2}\right)$ for all $e_{1}, e_{2} \in M_{G}$ with $d\left(e_{1}, e_{2}\right)=2$. This implies that for every edge $\tilde{e} \in \tilde{E}$ there are two points in $M_{G}$ at distance 1, such that every shortest path between these two points contains $\tilde{e}$. Thus:

Every $\tilde{e} \in \tilde{E}^{\prime \prime}$ lies on every shortest path from some $e \in E$ to $z$.

Lemma 3.4. $\sum_{\tilde{e} \in \tilde{E}^{\prime \prime}} \tilde{w}(\tilde{e}) \geq \frac{1}{2}|T|$.

Proof of Lemma 3.4. Let $\hat{H}(\hat{V}, \hat{E}, \tilde{w})$ be the connected component in subgraph $\tilde{H}^{\prime \prime}\left(\tilde{V}, \tilde{E}^{\prime \prime}, \tilde{w}\right)$ of $\tilde{H}$, which contains $z$.

Claim 1. $\hat{H}$ is a tree.

Proof of Claim 1. We indicate a bijection between $\hat{V}-\{z\}$ and $\hat{E}$ : let $\hat{e} \in \hat{E}$, $\hat{e}=\{x, y\}$. By (2) there is $e \in E$, such that every shortest path from $e$ to $z$ in $\hat{H}$ contains $\hat{e}$. Let $z-\cdots-x-y-\cdots-e$ be such a path. $\hat{e}$ is mapped on $y \in \hat{V}-\{z\}$. If both $\hat{e}_{1}, \hat{e}_{2} \in \hat{E}$ were mapped on the same $y \in \hat{V}-\{z\}$, one of $\hat{e}_{1}$ and $\hat{e}_{2}$ would be redundant, unless $\hat{e}_{1}=\hat{e}_{2}$. Thus, by the minimality of $\hat{H}$, the constructed mapping is injective. This yields $|\hat{E}| \leq|\hat{V}-\{z\}|$. Moreover, $|\hat{E}| \geq|\hat{V}-\{z\}|$ as $\hat{H}$ is connected. Therefore equality holds and $\hat{H}$ is a tree.

Claim 2. For every terminal vertex $x \in \hat{V}-\{z\}$ in $\hat{H} d_{\hat{H}}(x, z) \geq \frac{1}{2}$.

Proof of Claim 2. Case 1. $x \in E$. Hence $d_{\tilde{H}}(x, z)=1$ and therefore $d_{\hat{H}}(x, z) \geq 1 \geq \frac{1}{2}$.

Case 2. $x$ is an auxiliary vertex in $\tilde{H}$. When $\operatorname{deg} x \geqslant 3$, only one of these edges containing $x$ is in $\tilde{E}^{\prime \prime}$. Let $\tilde{e} \in \tilde{E}^{\prime}$ be another edge with endpoint $x$. $\tilde{e}$ lies on a shortest path from $e_{1}$ to $e_{2}$ for $e_{1}, e_{2} \in E, d\left(e_{1}, e_{2}\right)=1$. Without loss of generality $d_{\tilde{H}}\left(e_{1}, x\right) \leq \frac{1}{2}$,

$$
1=d_{\tilde{H}}\left(e_{1}, z\right) \leq d_{\tilde{H}}\left(e_{1}, x\right)+d_{\tilde{H}}(x, z) \leq \frac{1}{2}+d_{\tilde{H}}(x, z) .
$$

Hence $d_{\hat{H}}(x, z) \geq \frac{1}{2}$.

Claim 3. The set $\tilde{E}_{z}$ of all edges with endpoint $z$ in $\tilde{H}$ is in $\tilde{E}^{\prime \prime}$. Contrariwise assume $\{x, z\} \in \tilde{E}^{\prime}$. Thus there are $e_{1}, e_{2} \in E$ with distance 1 , such that $e_{1}-\cdots-x-$ $z-\cdots-e_{2}$ is a shortest path from $e_{1}$ to $e_{2}$ in $\tilde{H}$. Thus $1=d_{\tilde{H}}\left(e_{1}, e_{2}\right)>d_{\tilde{H}}\left(z, e_{2}\right)$, which is a contradiction.

Claims 1, 2, and 3 together yield

$$
\sum_{\tilde{\epsilon} \in \tilde{E}^{\prime \prime}} \tilde{w}(\tilde{e}) \geq \frac{1}{2} \operatorname{deg} \tilde{H} z .
$$

Claim 4.

$$
\operatorname{deg}_{\dot{H}} z \geq \min \left|T^{\prime}\right|, \quad T^{\prime} \text { transversal in } G .
$$

Proof of Claim 4. For $\tilde{e}=\{x, z\} \in \tilde{E}$ define

$$
E_{\tilde{e}}:=\{e \in E \mid \tilde{e} \text { lies on a shortest path from } z \text { to } e\} .
$$

Every pair $e_{1}, e_{2} \in E_{\tilde{e}}$ satisfies $d_{\tilde{H}}\left(e_{1}, x\right)<1, d_{\tilde{H}}\left(e_{2}, x\right)<1$. Therefore $d\left(e_{1}, e_{2}\right)=1$, and $e_{1}, e_{2}$ have a common endpoint $i$ in $G$. Thus $e_{1}=\{i, j\}, e_{2}=\{i, k\}$ for some $j, k \in V, j \neq k$. 
Pick another edge $e_{3} \in E_{\tilde{e}}$. $i \in e_{3}$, since otherwise $j \in e_{3}$ because of $d\left(e_{3}, e_{1}\right)=1$ and $k \in e_{3}$ because of $d\left(e_{3}, e_{2}\right)=1$, which yields a forbidden triangle $e_{1} e_{2} e_{3}$ in $G$. Put $i=v(\tilde{e})$, so $\{i\} \subseteq \bigcap_{e \in E_{\dot{e}}} e$.

Thus $E_{\tilde{e}} \subset\{e \mid i=v(\tilde{e})$ is an endpoint of $e$ in $G\}$. It remains to show that the set $v\left(\tilde{E}_{z}\right)=\left\{v(\tilde{e}) \mid \tilde{e} \in \tilde{E}_{z}\right\}$ is a transversal in $G$. But for any $e \in E$ there exists a path $z-x-\cdots-e$ of length 1 . Hence for the edge $\{z, x\}$ the associated vertex $v(\{z, x\})$ is both an endpoint of $e$ in $G$ and an element of $v\left(\tilde{E}_{z}\right)$. Altogether

$$
\sum_{\tilde{e} \in \tilde{E}^{n}} \tilde{w}(\tilde{e}) \underset{\langle(\})}{\gtrless} \frac{1}{2} \operatorname{deg}_{\tilde{H}} z \underset{(4)}{\geq} \frac{1}{2}|T|
$$

which proves Lemma 3.4 .

\section{Edge Lengths in Optimal Realizations}

In the last section we have shown that the optimal realization problem is NP-hard. In this section we will show that in the special case of integral metrics it is a member of NP. This follows from the fact that integral metrics have optimal realizations, in which all edge lengths are relatively simple fractionals. Together these two results will imply that our problem is NP-complete (for the NP-concept see [7]). Our first lemma deals with tree realizable metrics.

Lemma 4.1. Let $T(V, E, w)$ be a weighted tree without vertices of degree 2 and all pairs of terminal vertices having integral distance. Then $2 w(e) \in \mathbb{N}$ for all edges $e$ in $E$.

Proof. Follows trivially from analysis in [5], for instance.

For arbitrary optimal realizations such a simple result cannot hold. Nevertheless, Theorem 4.2 implies the NP-membership of the realization problem for integral metrics.

Theorem 4.2. Let $G(V, E, w)$ be a realization of an integral metric $(M, d)$ without redundant edges and with all auxiliary vertices having degree at least $3,|M|=m$. Then there exists a further edge weighting $\bar{w}: E \rightarrow \mathbb{R}^{\geq 0}$, such that $\bar{G}(V, E, \bar{w})$ is also a realization of $(M, d)$ and satisfies:

(a) Shortest paths between any two points of $M$ in $G$ remain shortest paths for these points in $\bar{G}$.

(b) $\sum_{e \in E} \bar{w}(e) \leq \sum_{e \in E} w(e)$.

(c) $\bar{w}(e)=p_{e} / q \in \mathbb{Q} ; p_{e}, q \in \mathbb{N} ; q \leq 2^{m^{10}}$ for all $e \in E$.

Before presenting the proof we want to illustrate Theorem 4.2 by a little example:

Let $G(V, E, w)$ be given by Fig. 11. Figure 12 shows the best possible choice of $\bar{w}$ to minimize $\sum_{e \in E} \bar{w}(e)$. One has $\sum_{e \in E} \bar{w}(e)=2,5 \leq 2+1 / \sqrt{3}=\sum_{e \in E} w(e)$, and $q=2$. 


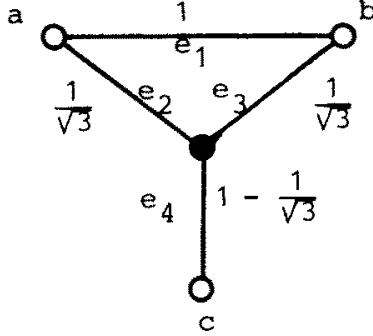

Fig. 11

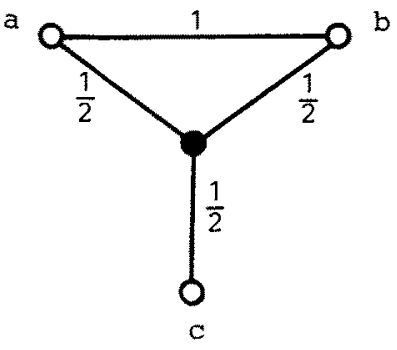

Fig. 12

Proof. $G$ does not contain redundant edges. Thus for every edge $e$ with an endpoint $z$ there exists some $i \in M$, such that every shortest path from $z$ to $i$ in $G$ begins with $e$. This implies $\operatorname{deg} z \leqslant m$ for all $z \in V$. Furthermore, $|V| \leq m^{4}$ by Remark 2.5. Altogether

$$
|E| \leq m^{5}
$$

For the further proof a linear program $L P(*)$ is defined:

1. Every edge $e \in E$ supplies a variable $x_{e}$.

2. Every path $i-e_{1}-\cdots-e_{r}-j$ of length $d(i, j)$ in $G$ between two points $i$, $j \in M$ supplies the $(=)$-constraint $x_{e_{1}}+\cdots+x_{e_{r}}=d(i, j)$.

3. Every simple path $i=e_{1}-\cdots-e_{\varsigma}-j$ of arbitrary length between two points $i, j \in M$ supplies the $(\geq)$-constraint $x_{e_{1}}+\cdots+x_{e_{i}} \geq d(i, j)$.

4. $x_{e} \geq 0$ for all $e \in E$.

5. There are no other constraints.

6. The objective function is $\min Z=x_{e_{1}}+\cdots+x_{e_{1}}$, where $E=\left\{e_{1}, \ldots, e_{t}\right\}$.

Considering the finiteness of $G$ and condition (5), LP(*) has only a finite number of constraints and at most $m^{5}$ variables. The set $K$ of feasible solutions of $L P(*)$ is nonemtpy, as $\left(w\left(e_{1}\right), \ldots, w\left(e_{t}\right)\right)$ is feasible. $G$ does not contain redundant edges, thus $0 \leq x_{e} \leq \max _{i, f \in M} d(i, j)$ for all $e \in E$. Hence $K$ is a compact convex polytope in $\mathbb{R}^{\prime}$.

In our example (Fig. 11) we obtain the following inequalities:

$$
\begin{aligned}
& x_{e_{1}} \quad=1, \\
& \begin{array}{llll}
x_{e_{2}} & & +x_{e_{4}} & =1, \\
& x_{e_{3}} & +x_{e_{4}} & =1,
\end{array} \\
& \begin{array}{llll}
x_{e_{1}} & +x_{e_{3}} & +x_{e_{4}} & \geq 1, \\
& & +x_{e_{3}} & \geq 1,
\end{array} \\
& x_{e_{1}}+x_{e_{2}}+x_{e_{4}} \geq 1 \\
& x_{e_{1}} \quad \geq 0 \text { for } i=1,2,3,4 \text {. }
\end{aligned}
$$

Claim. Every extremal point of $K$ has the form $\left(p_{1} / q, \ldots, p_{t} / q\right)$ with $p_{1}, \ldots, p_{t}$, $q \in \mathbb{N}$, and $q \leq 2^{m^{10}}$. 
Proof of the claim. For any extremal point $P$ of $K$ one can find $t$ linearly independent constrants of $\operatorname{LP}(*)$, which satisfy equality in $P$. Thus every extremal point of $K$ is the unique solution of a regular equation system

$$
A x=b \text {, }
$$

where $A$ is a $(t \times t)$-matrix with entries 0 and 1 only, $b \in \mathbb{N}^{\prime}$. (In all constraints of $L P(*)$ only the coefficients 0 and 1 occur on the left-hand side.)

In our example the first three and the fifth line constitute the matrix

$$
A=\left(\begin{array}{llll}
1 & 0 & 0 & 0 \\
0 & 1 & 0 & 1 \\
0 & 0 & 1 & 1 \\
0 & 1 & 1 & 0
\end{array}\right)
$$

Let $x^{*}=\left(x_{1}^{*}, \ldots, x_{t}^{*}\right)$ be a solution of (6). By Cramer's rule

$$
x_{i}^{*}=\frac{\operatorname{det}\left[\begin{array}{ccccc}
a_{11} & \cdots & b_{1} & \cdots & a_{1 t} \\
\vdots & & \vdots & & \vdots \\
a_{t 1} & \cdots & b_{t} & \cdots & a_{t t}
\end{array}\right]}{\operatorname{det}\left[\begin{array}{ccccc}
a_{11} & \cdots & a_{1 i} & \cdots & a_{11} \\
\vdots & & \vdots & & \vdots \\
a_{t 1} & \cdots & a_{t i} & \cdots & a_{t i}
\end{array}\right]} \text { for } i=1, \ldots, t
$$

Both determinants are integers. By Hadamard's inequality for determinants

$$
\operatorname{det} A \leq \prod_{j=1}^{t}\left(\sum_{i=1}^{t} a_{i j}^{2}\right)<\prod_{j=1}^{t} t=t^{t}, \quad t^{t} \leq 2^{t \log t} \leq 2^{t^{2}} \underset{(5)}{\leq} 2^{m^{10}} .
$$

An extremal optimal solution $\left(x_{1}, \ldots, x_{t}\right)$ of $L P(*)$ induces a weighting function $\bar{w}: E \rightarrow \mathbb{R}^{\geq 0}$ by $\bar{w}\left(e_{i}\right):=x_{i}$ for $i=1, \ldots, t . \bar{G}(V, E, \bar{w})$ satisfies conditions (a)-(c) of the theorem.

Remark 4.3. Let $d$ be an integral metric on $M=\{1, \ldots, m\}$. Theorem 4.2 supplies an optimal realization $G(V, E, w)$ of $(M, d)$ with $|V| \leq m^{4},|E| \leq m^{5}$, and $w\left(e_{i}\right)=$ $p_{i} / q, p_{i}, q \in \mathbb{N}, q \leq 2^{m^{10}}$. Thus this graph can be calculated by a nondeterministic algorithm in polynomial time relative to the input length of distance matrix $d[7]$.

The combination of NP-membership and NP-hardness yields that the realization problem is NP-complete. Thus it is a hopeless enterprise to search for a quick algorithm for the general realization problem.

In addition, we point out that the combinatorial dimension (a parameter introduced by Dress $\left[5\right.$, p. 380]) of the metrics $\left(M_{G}, d\right)$ constructed in Section 3 is at most two (this may be proved by a case-by-case consideration for any tuple of six points in $M_{G}$ ). Hence, while in the case of tree realizable metrics (i.e., for $\left.\operatorname{dim}_{\text {comb }}(M, d) \leq 1\right)$ the optimal realization problem is well known not to be NP-hard, it becomes NP-hard for spaces with $\operatorname{dim}_{\text {comb }}(M, d) \geq 2$. 


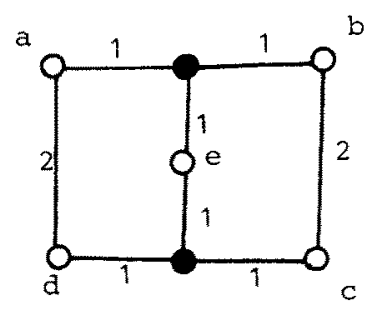

Fig. 13

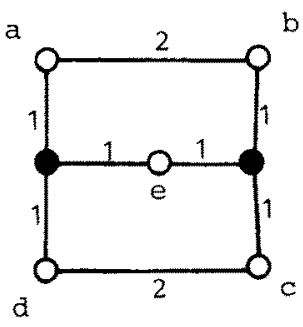

Fig. 14

\section{Examples of Optimal and Minimal Realizations}

5.1. Dress [5] gave the following example of a metric with two essentially different optimal realizations: $G_{1}\left(V_{1}, E_{1}, w_{1}\right)$ in Fig. 13 and $G_{2}\left(V_{2}, E_{2}, w_{2}\right)$ in Fig. 14 are different optimal realizations of the same metric on $M=\{a, b, c, d, e\}$. Dress conjectured that every metric $(M, d)$ has only a finite number of different optimal realizations. More precisely, he observed that, for any given unweighted graph $G(V, E)$ with $M \subset V$, the set of length-functions $w: E \rightarrow \mathbb{R}^{>0}$ which realize $(M, D)$ in an optimal fashion form a compact convex polytope, and he conjectured that this may always consist of one point only. Theorem 5.2 gives, as a counterexample, a metric with a continuum of optimal realizations, al with the same underlying unweighted graph.

Theorem 5.2. Every graph $G_{f}\left(V, E, w_{\varepsilon}\right)$ in Fig. 15 with $-\frac{1}{2} \leq \varepsilon \leq \frac{1}{2}$ is an optimal realization of the same metric $d$ on $M=\{a, b, c, d, e, f\}$.

Proof. $G_{e}$ realizes $(M, d)$ with $\sum_{e \in E} w_{F}(e)=9$ for all $-\frac{1}{2} \leq \varepsilon \leq \frac{1}{2}$. Hence it remains to show that, for every realization of $(M, d)$, the total edge length is at least 9 . Though there may be shorter proofs of this simple fact relying on some more general principles concerning optimal realizations, we find it instructive to prove this result in a rather straightforward way by exhaustive case-by-case considerations.

So, let $\bar{G}(\bar{V}, \bar{E}, \bar{w})$ be a realization of $(M, d)$,

$$
Q:=\{(a, b),(b, c),(c, d),(d, e),(e, f),(f, a)\}
$$

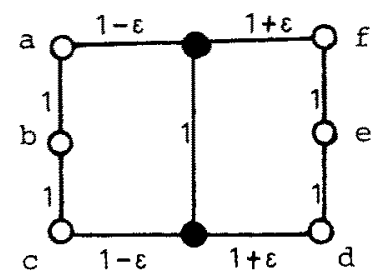

Fig. 15 
Claim 1. For every two distinct pairs $(i, j),(k, l) \in Q$ no vertex $v$ of a shortest path from $i$ to $j$ is an interior point of a shortest path from $k$ to $l$ in $\bar{G}$.

Proof of Claim 1. All cases are proved by contradiction.

Case 1: $(a, b),(b, c)$. Assume $v \in \bar{V}$ with $d_{\bar{G}}(a, v)=\varepsilon, d_{\vec{G}}(v, b)=1-\varepsilon$, $d_{\bar{G}}(v, c)=\delta, \quad 0 \leq \varepsilon \leq 1$, and $0<\delta<1$. Thus $d_{\bar{G}}(a, c) \leq d_{\bar{G}}(a, v)+d_{\bar{G}}(v, c)<2$. Contradiction.

Case 2: $(a, b),(c, d)$. Assume $v \in \bar{V}$ with $d_{\bar{G}}(a, v)=\varepsilon, d_{\bar{G}}(v, b)=1-\varepsilon$, $d_{\bar{G}}(c, v)=\delta, \quad d_{\bar{G}}(v, d)=2-\delta, \quad 0 \leq \varepsilon \leq 1, \quad 0 \leq \delta \leq 2, \quad$ and $0<\varepsilon+\delta<3$. Thus $d_{\bar{G}}(a, c)+d_{\bar{G}}(b, d) \leq d_{\bar{G}}(a, v)+d_{\bar{G}}(v, d)+d_{\bar{G}}(b, v)+d_{\bar{G}}(v, d)=3$

$<d(a, c)+d(b, d)=5$. Contradiction.

Case 3: $(a, b),(d, e)$. Assume $v \in \bar{V}$ with $d_{G}(a, v)=\varepsilon, d_{\bar{G}}(v, b)=1-\varepsilon$, $d_{\bar{G}}(d, v)=\delta, \quad d_{\bar{G}}(v, e)=1-\delta, \quad 0 \leq \varepsilon \leq 1, \quad 0 \leq \delta \leq 1$, and $0<\varepsilon+\delta<2$. Thus $d_{\bar{O}}(a, d)+d_{\tilde{G}}(b, e) \leq 2<d(a, d)+d(b, e)=7$. Contradiction.

Case 4: $(a, b),(e, f)$. Assume $v \in \tilde{V}$ with $d_{\bar{G}}(a, v)=\varepsilon, d_{\bar{G}}(v, b)=1-\varepsilon$, $d_{\overparen{O}}(e, v)=\delta, \quad d_{\bar{G}}(v, f)=1-\delta, \quad 0 \leq \varepsilon \leq 1, \quad 0 \leq \delta \leq 1, \quad$ and $0<\varepsilon+\delta<2$. Thus $d_{\bar{G}}(a, e)+d_{\bar{G}}(b, f) \leq 2<d(a, e)+d(b, f)=6$. Contradiction.

Case 5: $(a, b),(f, a)$. Assume $v \in \bar{V}$ with $d_{\bar{\sigma}}(a, v)=1-\varepsilon, d_{\bar{G}}(v, b)=\varepsilon$, $d_{\bar{\sigma}}(f, v)=\delta .0 \leq \varepsilon \leq 1,0 \leq \delta \leq 2$, and $0<\varepsilon+\delta<3$. Thus $d_{\bar{\sigma}}(b, f)<3=d(b, f)$. Contradiction.

Case 6: $(a, f),(c, d)$. Assume $v \in \bar{V}$ with $d_{\bar{G}}(a, v)=\varepsilon, d_{\bar{G}}(v, f)=2-\varepsilon$, $d_{\bar{G}}(c, v)=\delta, \quad d_{\bar{G}}(v, d)=2-\delta, \quad 0 \leq \varepsilon \leq 2, \quad 0 \leq \delta \leq 2$, and $0<\varepsilon+\delta<4$. Thus $d_{\bar{\sigma}}(a, d)+d_{\bar{G}}(c, f) \leq 4<d(a, d)+d(c, f)=6$. Contradiction.

Every other constellation of $(i, j),(k, l)$ is solved by one of these six cases with a symmetry argument. This proves Claim 1.

As a consequence of Claim 1, graph $\bar{G}_{1}$ in Fig. 16 is a subgraph of $\bar{G} . \bar{G}_{1}$ has a total edge iength of 8 . Theorem 5.2 holds if inserting a path with length 3 from $a$ to $d$ into $\bar{G}_{1}$ yields a total edge length $\geq 9$.

Claim 2. In $\bar{G}$, shortest paths from $a$ to $d$ and from $b$ to $c$ do not intersect.

Proof of Claim 2. Assume $v \in V$ with $d_{\bar{G}}(b, v)=\varepsilon, d_{\bar{\sigma}}(v, c)=1-\varepsilon, d_{\bar{G}}(a, v)=\delta$, $d_{\bar{G}}(v, d)=3-\delta, \quad 0 \leq \varepsilon \leq 1$, and $0 \leq \delta \leq 3$. Thus $d_{\bar{G}}(a, c)+d_{\bar{G}}(b, d) \leq 4<$ $d(a, c)+d(b, d)=5$. Contradiction.

By symmetry, Claim 2 is also valid for the pair $(a, d),(e, f)$.

Claim 3. In $\bar{G}$, a shortest path from $a$ to $d$ does not contain both an inner point of a shortest path from $a$ to $b$ and an inner point of a shortest path from $a$ to $f$.

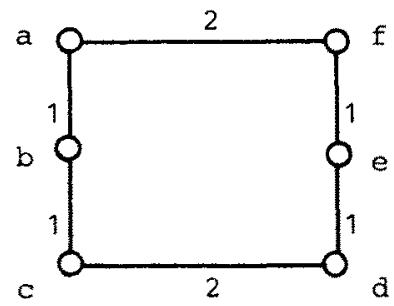

Fig. 16 


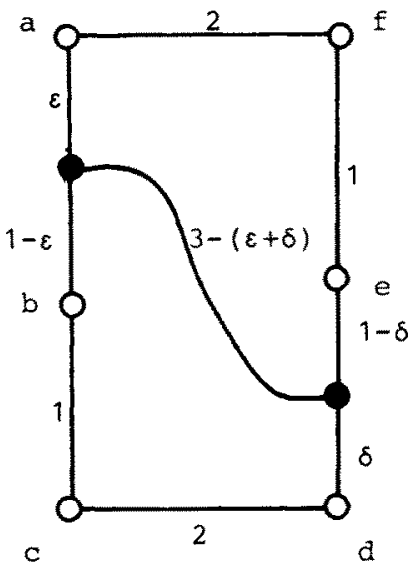

$\overline{\mathrm{G}}_{2}$

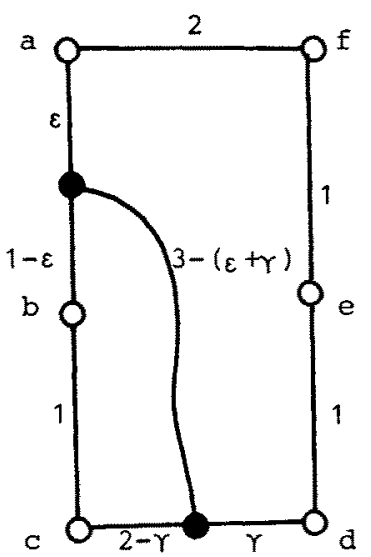

$\overline{\mathrm{G}}_{3}$

Fig. 17

Fig. 18

Proof of Claim 3, Assume a shortest path $a-\cdots-v-\cdots-w-\cdots-d$ from $a$ to $d$, where $v$ is an inner point of a shortest path from $a$ to $b$ and $w$ is an inner point of a shortest path from $a$ to $f . d_{\bar{G}}(b, f)=d_{\bar{G}}(b, a)+d_{\bar{G}}(a, f)$ forces $d_{\bar{G}}(v, w)=d_{\bar{G}}(v, a)+d_{\bar{G}}(a, w)$. Thus $d_{\bar{G}}(a, w)=d_{\bar{G}}(a, v)+d_{\bar{G}}(v, w)=d_{\bar{G}}(a, v)+$ $d_{\bar{G}}(v, a)+d_{\bar{G}}(a, w)>d_{\bar{G}}(a, w)$. Contradiction. The same idea solves the other case with $v$ between $a$ and $f$, and $w$ between $a$ and $b$.

Symmetry makes Claim 3 also valid for the triple $(a, d),(d, c),(d, e)$.

By Claims 2 and 3, $\bar{G}$ contains one of the four graphs in Figs. 17-20 as a subgraph.

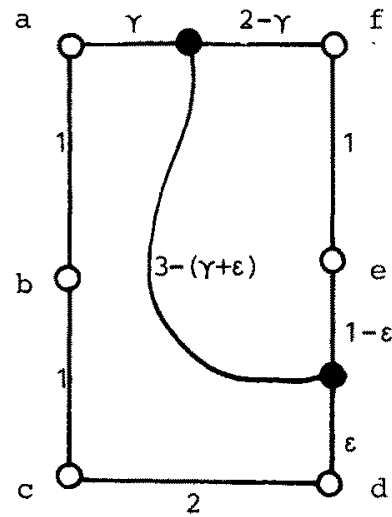

$\bar{G}_{4}$

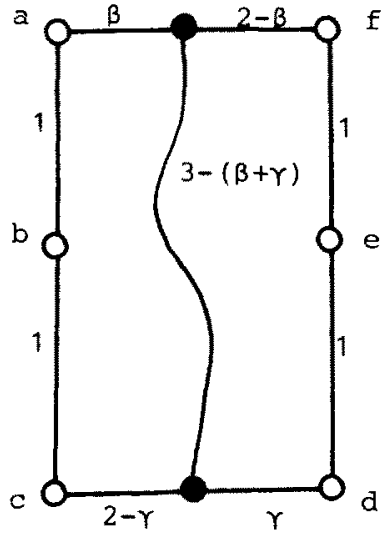

$\overline{\mathrm{G}}_{5}$

Fig. 20

Fig. 19 
Case 1. $\bar{G}_{2} \subset \bar{G}$ with $0 \leq \varepsilon \leq 1,0 \leq \delta \leq 1$.

$$
4=d_{\bar{\sigma}}(b, e) \leq(1-\varepsilon)+3-(\varepsilon+\delta)+(1-\delta)=5-2(\varepsilon+\delta) .
$$

Thus $\varepsilon+\delta \leq \frac{1}{2}$ and $3-(\varepsilon+\delta) \geq \frac{5}{2}$. Therefore

$$
\sum_{e \in E} \bar{w}(e) \geq 8+\frac{5}{2}>9 .
$$

Case 2. $\bar{G}_{3} \subset \bar{G}$ with $0 \leq \varepsilon \leq 1,0 \leq \gamma \leq 2$.

$$
\begin{aligned}
& 3=d_{\bar{G}}(b, d) \leq(1-\varepsilon)+3-(\varepsilon+\gamma)+\gamma=4-2 \varepsilon, \\
& 2=d_{G}(a, c) \leq \varepsilon+3-(\varepsilon+\gamma)+(2-\gamma)=5-2 \gamma, \quad \text { thus } \quad \varepsilon \leq \frac{1}{2} .
\end{aligned}
$$

By (7) and $(8), 3-(\varepsilon+\gamma) \geq 1$, which yields

$$
\sum_{e \in \bar{E}} \bar{w}(e) \geq 8+1=9 .
$$

Case 3. $\bar{G}_{4} \subset \bar{G}$ yields a total edge length $\geq 9$ by the same arguments as in Case 2.

Case 4. $\bar{G}_{5} \subset \bar{G}$ with $0 \leq \beta \leq 2,0 \leq \gamma \leq 2$.

$$
3=d_{G}(c, f) \leq(2-\beta)+3-(\beta+\gamma)+(2-\gamma)=7-2(\gamma+\beta)
$$

Thus $\gamma+\beta \leq 2$, and $3-(\beta+\gamma) \geq 1$. Therefore

$$
\sum_{e \in \bar{E}} \bar{w}(e) \geq 8+1=9 .
$$

Dress [5] defined hereditarily optimal realizations $G(V, E, w)$ of finite metric spaces $(M, d)$ by induction: if $|M| \leq 2$, then any optimal realization of $(M, d)$ is defined to be hereditarily optimal. If $|M|=k$ and if hereditarily optimal realizations have been defined already for all metric spaces $(\bar{M}, d)$ with $|\bar{M}|<k$, then a realization $H=H(V, E, w)$ of $(M, d)$ is defined to be hereditarily optimal if for any $\bar{M} \varsubsetneqq M$ there is some subgraph $\bar{H}$ of $H$ such that $\bar{H}$ is a hereditarily optimal realization of $\left(\bar{M}, d_{\mid \bar{M}}\right)$, and if $\sum_{E \in E} w(e)$ is minimal with respect to this property.

Dress [5] has shown that hereditarily optimal realizations are unique up to isomorphy.

The above disproved conjecture of Dress was hoped to be a corollary of the much stronger conjecture that any optimal realization $G$ of a finite metric space $(M, d)$ can be derived from its hereditarily optimal realization $H=H(M, d)$ by "merely" deleting some edges of $H$ (though it may be NP-hard to decide which edges to delete). But while the above example disproves this conjecture as well, it suggests a possibly positive answer to the following interesting new question, relating optimal and hereditarily optimal realizations of finite metric spaces in a more subtle way: given a finite metric space $(M, d)$ and a finite undirected simple 


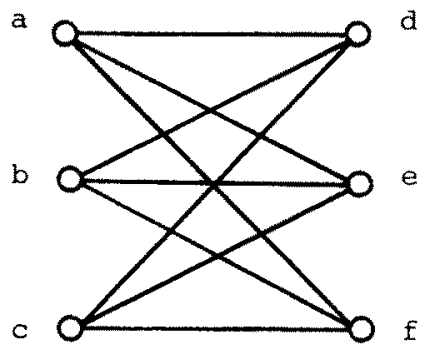

Fig. 21

graph $\left(V, E \subseteq\left(\begin{array}{l}V \\ 2\end{array}\right)\right)$ with $M \subseteq V$, consider the (possibly empty) set $W=$ $(M, d ; V, E)$ of all weight functions $w: E \rightarrow \mathbb{R}^{20}$ which make $G=G(V, E, w)$ an optimal realization of $(M, d)$. Noting that $W$ is a compact convex polytope in $\mathbb{R}^{E}$ one may ask whether at least its extremals can be derived from $H(M, d)$ "merely" by edge deletion (in the above example $W$ is an interval and its two endpoints can indeed be derived in that way). Of course, to decide which edges are to be deleted might again be NP-hard, but at least a positive answer to this

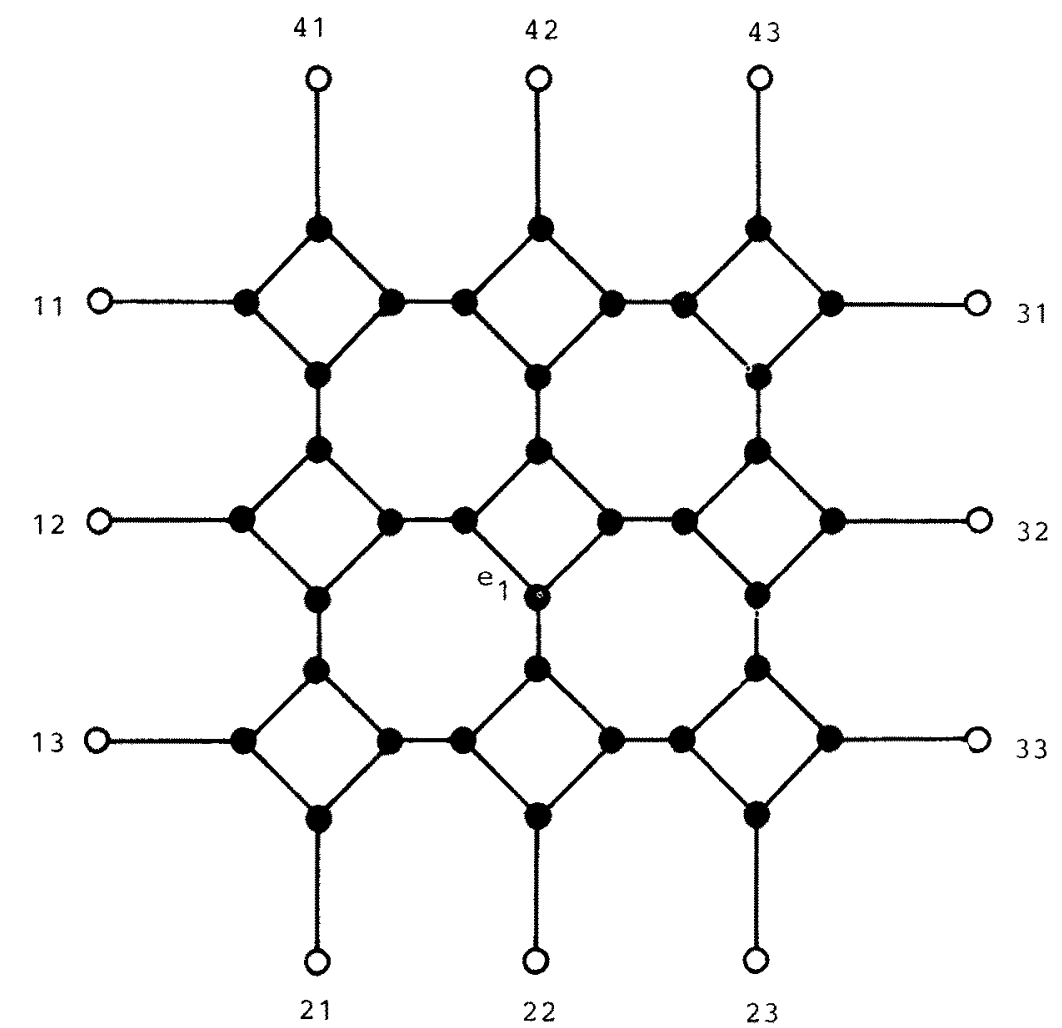

Fig. 22 
question would improve the result of Theorem 4.2(c) impressively, since in hereditarily optimal realizations of metric spaces with only integral distances all edge lengths are integral multiples of $\frac{1}{2}$ (proof in [5]).

Remark 5.3. Optimal realizations of metrics need not be planar. The graph in Fig. 21 with all edges having length 1 is the unique optimal realization of the underlying metric on $M=\{a, b, c, d, e, f\}$. The optimality may be proved by Theorem C.

5.4. By Remark 2.5 minimal realizations of a metric $(M, d)$ contain at most $|M|^{4}$ auxiliary vertices. For $n \geq 1$ there exist metrics $(M, d)$ and minimal realizations $G(V, E, w)$ with $|M|=4 n,|V|=4 n^{2}+4 n$, that means $G$ has quadratically many auxiliary vertices. Figure 22 shows such a graph $G$ for $n=3$. All edges have length $1,(M, d)$ with $M=\{11,12,13,21,22,23,31,32,33,41,42,43\}$ is the metric induced by $G$. $e_{1}$ lies on the unique shortest path from 11 to 23 .

\section{Extremal (Metric, Optimal Realization) Pairs}

For every metric $(M, d)$ with $M=\{1, \ldots, m\}$ define

$$
Q(M, d):=\frac{\sum_{e \in E} w(e)}{\sum_{1 \leq i<j \leq m} d(i, j)},
$$

where $G(V, E, w)$ is an optimal realization of $(M, d)$. Extremal values of $Q(M, d)$ for certain classes of metrics yield necessary conditions for the corresponding optimal realizations:

$$
\begin{aligned}
& p_{m}:=\inf _{|M|=m} Q(M, d), \\
& q_{m}:=\sup _{|M|=m} Q(M, d) .
\end{aligned}
$$

For $m=2, p_{m}-q_{m}=1$.

Theorem 6.1.

$$
\begin{gathered}
p_{m}=\left\{\begin{array}{l}
\frac{4}{m^{2}} \quad \text { if } m \text { is even, } \quad m \geq 2, \\
\frac{4}{m^{2}-1} \quad \text { if } m \text { is odd, } \quad m \geq 3 .
\end{array}\right. \\
\quad \frac{1}{3}<q_{m} \leq 1 \quad \text { for all } \quad m \geq 2 .
\end{gathered}
$$

Proof.

ad $p_{m}$. Let $(M, d)$ be a metric and $G(V, E, w)$ an optimal realization of $(M, d)$, $M=\{1, \ldots, m\}$. For every edge $e$

$$
U_{e}:=\{\{i, j\} \subset M \mid e \text { lies on a shortest path from } i \text { to } j\} .
$$




\section{Claim.}

$$
\left|U_{\{x, y\}}\right| \leq \begin{cases}\left(\frac{m}{2}\right)^{2} & \text { if } m \text { is even } \\ \frac{(m-1)}{2} \frac{(m+1)}{2} & \text { if } m \text { is odd }\end{cases}
$$

for every edge $\{x, y\} \in E$.

Proof of the claim. $M$ is divided in two subsets by

$$
\begin{aligned}
& M_{1}:=\left\{i \in M \mid d_{G}(i, x) \leq d_{G}(i, y)\right\}, \\
& M_{2}:=M-M_{1} .
\end{aligned}
$$

For $\{i, j\} \in U_{\{x, y\}}$ either $i \in M_{1}, j \in M_{2}$ or $j \in M_{1}, i \in M_{2}$. Thus

$$
\left|U_{\{x, y\}}\right| \leq\left|M_{1}\right| \cdot\left|M_{2}\right| \leq \begin{cases}\left(\frac{m}{2}\right)^{2} & \text { if } m \text { is even, } \\ \frac{(m-1)}{2} \frac{(m+1)}{2} & \text { if } m \text { is odd, }\end{cases}
$$

and the claim holds.

For every pair $i, j \in M$ let

$$
E_{i, j}:=\{e \in E \mid e \text { lies on a shortest path from } i \text { to } j\}
$$

With this notation

$$
\begin{aligned}
& Q(M, d)=\frac{\sum_{e \in E} w(e)}{\sum_{1 \leq i<j \leqslant m} d(i, j)} \geq \frac{\sum_{e \in E} w(e)}{\sum_{1 \leq i<j \leq m}\left[\sum_{e \in E_{1, j}} w(e)\right]} \\
& =\frac{\sum_{e \in E} w(e)}{\sum_{e \in E[}\left[\sum_{\substack{\{i, j\} \in U_{e} \\
\text { with } i<j}} w(e)\right]}=\frac{\sum_{e \in E} w(e)}{\sum_{e \in E} w(e)|U(e)|} \\
& \underset{(10)}{\gtrless} \begin{cases}\frac{4}{m^{2}} & \text { if } m \text { is even, } \\
\frac{4}{m^{2}-1} & \text { if } m \text { is odd. }\end{cases}
\end{aligned}
$$




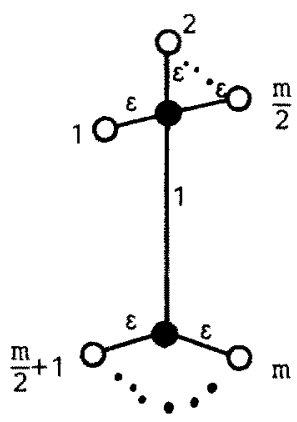

Fig. 23

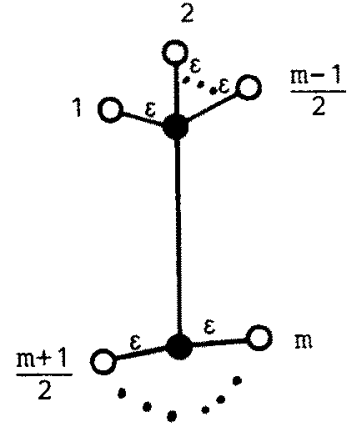

Fig. 24

Thus $4 / m^{2}$ and $4 /\left(m^{2}-1\right)$ are lower bounds for $Q(M, d)$ with $m$ even or odd, respectively. The optimality of these bounds is shown by the following metrics. Let $m \geq 2$. For $\varepsilon>0$,

$$
d_{\varepsilon}(i, j):= \begin{cases}2 \varepsilon & \text { if } i \leq m / 2, j \leq m / 2 \\ 2 \varepsilon & \text { if } i>m / 2, j>m / 2 \\ 1+2 \varepsilon & \text { otherwise. }\end{cases}
$$

The optimal realizations of $\left(M, d_{\varepsilon}\right)$ are given by Fig. 23 if $m$ is even and by Fig. 24 if $m$ is odd. (Proof by Theorem D.)

$$
\lim _{\varepsilon \rightarrow 0} Q\left(M, d_{\varepsilon}\right)= \begin{cases}\frac{4}{m^{2}} & \text { if } m \text { is even, } \\ \frac{4}{m^{2}-1} & \text { if } m \text { is odd }\end{cases}
$$

ad $q_{n} . \quad Q(M, d) \leq 1$ for all metrics $(M, d)$ with $|M| \geq 2$, since an optimal realization does not contain redundant edges. $Q(M, d)>\frac{1}{3}$ is given in the following examples.

Case 1. $m$ is even, $m \geq 2$. The complete bipartitie graph $K_{m / 2, m / 2}$ with all edges having length 1 realizes the induced metric on its vertices, optimality by Theorem C. Thus

$$
Q(M, d)=\frac{(m / 2)^{2}}{(m / 2)^{2}+2 \cdot 2\left(\begin{array}{c}
m / 2 \\
2
\end{array}\right)}=\frac{1}{3-4 / m}
$$

Case 2. $m$ is odd, $m \geq 3$. The complete bipartite graph $K_{(m+1) / 2,(m-1) / 2}$ with all edges having length 1 realizes the induced metric on its vertices, optimality by 
Theorem C. Thus

$$
Q(M, d)=\frac{((m+1) / 2)((m-1) / 2)}{\left(\frac{m+1}{2}\right)\left(\frac{m-1}{2}\right)+2\left(\begin{array}{c}
(m+1) / 2 \\
2
\end{array}\right)+2\left(\begin{array}{c}
(m-1) / 2 \\
2
\end{array}\right)}=\frac{1+1 / m}{3-1 / m}
$$

6.2. Open Question. Is

$$
q_{m}= \begin{cases}\frac{1}{3-4 / m} & \text { for even } m ? \\ \frac{1+1 / m}{3-1 / m} & \text { for odd } m ?\end{cases}
$$

\section{References}

1. F. T. Boesch, Properties of the distance matrix of a tree, Quart. Appl. Math. 26 (1968), 607-609.

2. P. Buneman, A note on the metric properties of trees, J. Combin. Theory Ser. B 17 (1974), 48-50.

3. S. Chaiken, A. K. Dewdney, and P. S. Slater, An optmal diagonal tree code, SIAM J. Algebraic Discrete Methods 4 (1983), 42-49.

4. A.K. Dewdney, Diagonal tree codes, Inform. and Control 40 (1979), 234-239.

5. A. W. M. Dress, Trees, tight extensions of metric spaces, and the cohomological dimension of certain groups: a note on combinatorial properties of metric spaces, Adv, in Math. 53 (1984), $321-402$

6. M. Eigen, W. Gardiner, P. Shuster and R. Winkler-Oswatitsch, The origin of genetic information, Sci. Amer. 244 (1981), 78-94.

7. M. R. Garey and D. S. Johnson, Computers and Intractability, a Guide to the Theory of NPCompleteness, Freeman, San Francisco, 1979.

8. S. L. Hakimi, Optimum locations of switching centers and medians of a graph, Oper. Res. 12 $(1964), 450-459$.

9. S. L. Hakimi and S. S. Yau, Distance matrix of a graph and its realizability, Quart. Appl. Math. 22 (1964), 305-317.

10. W. Imrich, Realisierung von Metriken in Graphen, Österreich. Akad. Wiss. Math.-Natur. Kl. Sitzungsber. /I 178 (1969), 19-24.

11. W. Imrich, On metric properties of tree like spaces, in Contributions to Graph Theory and Its Applications (Intern. Colloq. Oberhof 1977) 129-156, Technische Hochschule Ilmenau, Ilmenau, 1977.

12. W. Imrich and G. Schwarz, Trees and length functions in groups, Ann. Discrete Math. 17 (1983), 347-359.

13. W. Imrich and E. Stockii, On optimal embeddings of metrics in graphs, Sibirsk. Mat. Z. 13 (1972), $558-565$.

14. W. Imrich, J. M. S. Simões-Pereira, and C. M. Zamfirescu, On optimal embeddings of metrics in graphs, J. Combin. Theory Ser. B 36 (1984), 1-15.

15. A. N. Patrinos and S. L. Hakimi, The distance matrix of a graph and its tree realization, Quart. Appl. Math. 30 (1972) 255-269.

16. P. H. Sellers, The theory and computation of evolutionary distances: pattern recognition, $J$. Algorithms I (1980), 359-373.

17. J. M. Simões-Pereira, A note on the tree realizability of a distance matrix, $J_{2}$ Combin. Theory 6 (1969), 303-310.

18. J. M. S. Simóes-Pereira, A note on optimal and suboptimal digraph realizations of quasidistance matrices, SIAM J. Algebraic Discrete Methods 5 (1984), 117-132. 
19. J. M. S. Simöes-Pereira and C. M. Zamfirescu, Submatrices of non-tree-realizable distance matrices, Linear Algebra Appl. 44 (1982), 1-17.

20. E. A. Smolenskii, A method for the linear recording of graphs, Zh. Vychisl. Mat. $i$ Mat. Fiz. 2 (1962), 371-372 (in Russian).

21. B. C. Tansel, R. L. Francis, T. J. Lowe, and M. L. Chen, Duality and distance constraints for the non-linear p-center problem and covering problem on a tree network, Oper. Res. 30 (1982), 725-744.

22. K. A. Zareckii, Constructing a tree on the basis of a set of distances between the hanging vertices, Uspekhi Mat. Nauk 20 (1965), 90-92 (in Russian).

Received February 10, 1986, and in revised form October 30, 1986. 